\title{
ESSENTIAL MAPS AND EMBEDDINGS OF ANNULI IN NONORIENTABLE $M^{3}$
}

\author{
R. J. DAIGLE AND C. D. FEUSTEL ${ }^{1}$
}

ABSTRACT. In this note we give an example which shows that the exi stence of an essential map of an annulus in a nonorientable 3-manifold does not guarantee the existence of an essential embedding in that manifold.

I. Introduction. It has been reported by F. Waldhausen that the existence of an "essential" map of an annulus into a compact orientable 3manifold guarantees the existence of an essential embedding of an annulus in that 3-manifold. It is natural to ask if the result above is true for nonorientable 3-manifolds. In this note we give an example to show that the answer to the question above is in general no.

Feustel has proved [2] that the existence of a certain type of essential map of an annulus into a compact (not necessarily orientable) 3-manifold guarantees the existence of an embedding having the same properties. Our example also shows that Feustel's conditions are necessary in case the 3-manifold is not orientable.

II. Notation. We shall let $A$ denote an annulus, $F$ a mobius band, $S^{1}$ the 1-sphere, and $M$ a 3-manifold. All spaces will be simplicial complexes and all maps will be piecewise linear. We shall denote the boundary of a manifold $N$ by $\partial N$ and the components of $\partial A$ by $c_{1}$ and $c_{2}$. We let $\alpha$ be an arc embedded in $A$ such that $c_{j} \cap \alpha$ is an endpoint of $\alpha$ for $j=$ 1, 2. Such an arc is a spanning arc of $A$. A map $f:(A, \partial A) \rightarrow(M, \partial M)$ is essential if

(1) $f_{*}: \pi_{1}(A) \rightarrow \pi_{1}(M)$ is monic,

(2) the arc $f(\alpha)$ is not homotopic rel its boundary to an arc in $\partial M$. We remark that condition (2) above is independent of our choice of the

Received by the editors May 8, 1973.

AMS (MOS) subject classifications (1970). Primary 55A25; Secondary 57C 35. Key words and phrases. Essential map, essential embedding.

1 This author is partially supported by NSF Grant GP 15357. 
spanning arc a. A connected surface $S$ embedded in a manifold $M$ is in. compressible in $M$ if $S$ is not the 2 -sphere and the natural map $i_{*}: \pi_{1}(S) \rightarrow$ $\pi_{1}(M)$ induced by inclusion is monic.

We shall say that a simple loop $\lambda_{1}$ in the interior of $F \times[0,1]$ is knotted in $F \times[0,1]$ if the complement of the interior of a regular neighborhood $R$ of $\lambda_{1}$ in $F \times[0,1]$ is not homeomorphic to the product to a klein bottle with $[0,1]$. Let $\lambda$ be a 1-sided simple loop in the interior of $F$ and $t$ a point in $[0,1]$. One can find a knotted loop $\lambda_{1}$ in $F \times[0,1]$ by altering the loop $\lambda \times\{t\}$ inside a regular neighborhood of one of the points on $\lambda \times\{t\}$.

Let $F_{1}$ and $F_{2}$ be surfaces in $M$ or $\partial M$. Then $F_{1}$ is parallel to $F_{2}$ if and only if there exists an embedding of $F_{1} \times[0,1]$ in $M$ such that $F_{1}=$ $F_{1} \times\{0\}$, and $F_{2}$ is the closure of $\partial\left(F_{1} \times[0,1]\right)-F_{1} \times\{0\}$.

III. Construction of the manifold $M$. Let $x$ be a point in $S^{1}$ and $M_{1}=$ $F \times S^{1}$. Let $R$ be a regular neighborhood of $\lambda \times\{x\}$ in $M_{1}$. Then $R$ is homeomorphic to $F \times[0,1]$. Let $\mathscr{D}$ be a disk embedded in $R$ such that $\mathscr{D} \cap$ $\partial R=\partial \mathscr{D}$ and $R-\mathscr{D}$ is connected. Let $\lambda_{1}$ be a knotted loop in $R$ such that $\lambda_{1}$ meets $\mathscr{D}$ in a single point and crosses $\mathscr{D}$ at that point. Let $R_{1}$ be a regular neighborhood of $\lambda_{1}$ in $R$. Denote $\partial R_{1}$ by $K_{1}$ and $\partial R$ by $K$. Let $M_{2}^{1}$ be the 3-manifold obtained by removing the interior of $R_{1}$ from $M_{1}$. Let $h: M_{2}^{1} \rightarrow M_{2}^{2}$ be a homeomorphism and $M$ the union of $M_{2}^{1}$ and $M_{2}^{2}$ with the identification $y=h(y)$ for $y \in K_{1}$.

IV. There is an essential map of $A$ into $M$. Let $p:(A, \partial A) \rightarrow(F, \partial F)$ be a covering map. Let $x_{0}$ be a point in $S^{1}$ such that $R$ does not meet $F \times\left\{x_{0}\right\} \subset M_{1}$. Then we may define $f:(A, \partial A) \rightarrow\left(M_{2}^{1}, \partial M_{2}^{1}\right)$ by $f(s)=$ $\left(p(s), x_{0}\right)$. We claim that $f$ is essential. Clearly condition (1) is satisfied since the natural map $i_{*}: \pi_{1}\left(M_{2}^{1}\right) \rightarrow \pi_{1}(M)$ induced by inclusion is monic.

Let $(\hat{M}, \hat{p})$ be the orientable double cover of $M$. Now there is a map $\hat{f}: A \rightarrow \hat{M}$ such that $\hat{p} \hat{f}=f$. It can be seen that $\hat{f}\left(c_{1}\right)$ and $\hat{f}\left(c_{2}\right)$ lie on different components of $\partial \hat{M}$. It follows that $f(\alpha)$ is not homotopic rel its boundary to an arc in $\partial M$ since such a homotopy could be lifted to $\hat{M}$.

$V$. There are no essential embeddings of $A$ in $M$. Suppose that $g$ : $(A, \partial A) \rightarrow(M, \partial M)$ is an essential embedding. We claim that we may assume that $g(A)$ does not meet $K_{1}$. After a general position argument, we may assume that $g^{-1}\left(K_{1}\right)$ is a collection (possibly empty) of simple closed loops. Since $K_{1}$ and $g(A)$ are incompressible in $M$, any loop in $K_{1} \cap g(A)$, nullhomotopic in $M$, is nullhomotopic both on $g(A)$ and on $K_{1}$. It follows from 
standard arguments that we may suppose that no loop in $K_{1} \cap g(A)$ is nullhomotopic in $M$. Similarly we may suppose that $K \cap g(A)$ and $h(K) \cap g(A)$ are collections of simple loops and that no loop in either collection is nullhomotopic in $M$. We suppose that $g(A)$ meets $K_{1}$.

Note that up to isotopy, the only simple essential loop on $T=\partial M_{2}^{1} \cap \partial M$ which is homologous to a loop on $K$ is $\partial F \times\left\{x_{0}\right\}$. We may assume that $g(A)$ meets $T$ and that there is an annulus $A_{1} \subset g(A)$ such that $A_{1} \cap(T \cup K)=$ $\partial A_{1}$. Thus $\partial A_{1} \cap T$ is twice the generator of $\pi_{1}(R)$. Let $A_{2} \subset g(A)$ be an annulus such that $A_{2} \cap\left(K \cup K_{1}\right)=\partial A_{2}$ and $A_{2}$ meets both $K_{1}$ and $K$. Since $\partial A_{1} \cap \partial A_{2} \cap K$ is either empty or all of $\partial A_{1} \cap K$ and $\partial A_{1}$ is orientable, standard arguments will show that $\lambda_{1}$ must have been unknotted. It follows that we may assume that $g(A)$ does not meet $K_{1}$ and that $g(\partial A) \subset$ $\partial M_{2}^{1}$.

It is now clear that we may regard $g$ as an essential embedding of $A$ in $M_{2}^{1}$ or simply $M_{1}-\lambda_{1}$. Let $(\hat{M}, \hat{p})$ be the orientable double cover of $M_{1}$. Since $g(A) \subset M_{1}-\lambda_{1}$ and $\left[g\left(c_{1}\right)\right] \in \hat{p}_{*} \pi_{1}\left(\hat{M}_{1}\right)$, we can find a map $\hat{g}: A \rightarrow$ $\hat{M}_{1}$ such that $\hat{p} \hat{g}=g$ and $\hat{g}(A) \subset \hat{M}_{1}-\hat{p}^{-1} \lambda_{1}$. Suppose that $\hat{g}(\partial A)$ lies on a single component of $\partial \hat{M}_{1}$. Let $\rho: \hat{M}_{1} \rightarrow \hat{M}_{1}$ be the nontrivial covering translation of $\hat{M}_{1}$. Since $\hat{M}_{1}$ is homeomorphic to the product of a torus $T$ with $[0,1]$, it will follow from [3, Corollary 3.2] that $\hat{g}(A)$ and $\rho \hat{g}(A)$ are parallel to annuli in $\partial \hat{M}_{1}$. Since $\hat{p}^{-1} \lambda_{1}$ is connected and $\hat{g}(\partial A)$ and $\rho \hat{g}(\partial A)$ lie on distinct components of $\partial \hat{M}_{1}$, it will follow that either $\hat{g}(\alpha)$ or $\rho \hat{g}(\alpha)$ is homotopic rel its boundary to an arc in $\partial \hat{M}_{1}$ in the complement of $\hat{p}^{-1} \lambda_{1}$. One can project this homotopy to show that $g(A)$ was not essential.

Suppose that $\hat{g}$ carries $c_{1}$ and $c_{2}$ to distinct components of $\partial \hat{M}_{1}$. We observe that $\hat{g}(A) \cap \rho \hat{g}(A)$ is empty since $g$ is an embedding. Let $N_{1}$ and $N_{2}$ be the closures of the components of the complement of $\hat{M}_{1}-(\hat{g}(A) \cup \rho \hat{g}(A))$. Then we may suppose that $\hat{p}^{-1} \lambda_{1} \subset N_{1}$ since $\hat{p}^{-1}\left(\lambda_{1}\right)$ is connected. We claim that $\rho N_{1}=N_{1}$. Suppose that there is a point $z \in\left(N_{1}-\rho N_{1}\right)$. Then $\rho z \in N_{2}$ and we can find a path $\beta$ in $N_{1}$ from $z$ to $\lambda_{1}$ which does not meet $\hat{g}(A) \cup$ $\rho \hat{g}(A)$. But now $\rho \beta$ is a path which does not meet $\hat{g}(A) \cup \rho \hat{g}(A)$. It follows that $\rho \beta \subset N_{2}$ and $\hat{p}^{-1}\left(\lambda_{1}\right)$ meets $N_{2}$. This is impossible. Since $\rho\left(N_{1}-\rho N_{1}\right)=$ $\rho N_{1}-N_{1}, N_{1}=\rho N_{1}$. Similarly $\rho N_{2}=N_{2}$.

It is now clear that $g(A)$ separates $M_{1}$ into two components whose closures are $X_{1}=p N_{1}$ and $X_{2}=p N_{2}$. We obtain the commutative diagram shown in Figure 1 where all homomorphisms are induced by inclusion. Since $\pi_{1}\left(M_{1}\right)=z \oplus z, \pi_{1}\left(M_{1}\right)$ is not a nontrivial free product with amalgamation. Thus $i_{1}$ or $i_{2}$ must be onto. Since $\lambda_{1} \subset X_{1}, i_{2}$ is onto. It follows from 


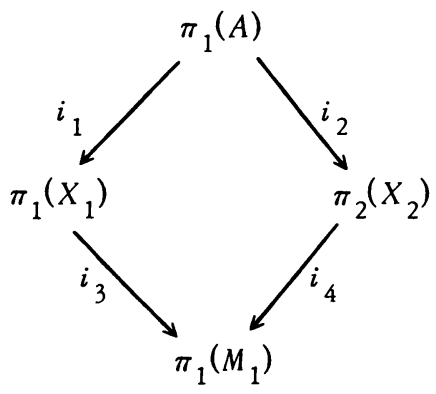

Figure 1

[1, Theorem (1.1)] that $X_{2}$ is homeomorphic to $A \times[0,1]$ and that $g(a)$ is homotopic in $X_{2}$ rel its boundary to an arc in $\partial M_{1}$.

Thus $g$ was not essential.

\section{REFERENCES}

1. E. M. Brown and R. H. Crowell, Deformation retractions of 3-manifolds into their boundaries, Ann. of Math. (2) 82 (1965), 445-458. MR 31 \#6236.

2. C. D. Feu stel, On embedding essential annuli in $M^{3}$, Canad. J. Math. (to appear).

3. F. Waldhausen, On irreducible 3-manifolds which are sufficiently large, Ann. of Math. (2) 87 (1968), 56-88. MR $36 \# 7146$.

DEPARTMENT OF MATHEMATICS, VIRGINIA POLYTECHNIC INSTITUTE AND STATE UNIVERSITY, BLACKSBURG, VIRGINIA 24061 